\title{
Research on Guangxi Gaoshan Han Folk Songs from the Perspective of Ethnic Identity
}

\author{
Zhang Chengjia ${ }^{1, a}$ \\ ${ }^{1}$ School of Music and Performing Arts, Sichuan University of Arts and Science, Tongchuan District, Dazhou City, \\ Sichuan Province, China \\ a984116070@qq.com
}

\begin{abstract}
Han folk songs in Gaoshan in northwestern Guangxi are mainly popular among the Han people in Leye County, Tianlin County, Longlin County and Lingyun County under the jurisdiction of Baise City, Guangxi. It also attracts the love of many other ethnic minorities. The Han people of Gaoshan come from different places, but they have formed the same music culture and integrate folk songs perfectly into their lives. This is directly related to the strong and continuous sense of identity of the Han people of Gaoshan with their own national culture. From the perspective of the social function and ethnic cultural identity of Gaoshan Han folk songs, this article analyzes the development status of Gaoshan Han folk songs in northwest Guangxi.
\end{abstract}

Keywords: ethnic identity, Gaoshan Han in Guangxi, folk songs, cultural identity

\section{基于族群认同视域下的广西高山汉民歌研究}

\author{
张承嘉 $1, \mathrm{a}$
}

1 四川文理学院音乐与演艺学院, 通川区, 达州市, 四川省, 中国 a984116070@qq.com

\section{摘要}

桂西北高山汉民歌主要流行在广西百色市下辖的乐业县、田林县、隆林县以及凌云县的汉族中，同时也吸引了 许多其他少数民族人民的喜爱。高山汉人民来自不同的地方，但却形成相同的音乐文化，并将民歌完美地融入 生活中，与高山汉人民对本民族文化有强烈、持续的认同感有直接的关系。本文将从高山汉民歌的社会功能、 族群文化认同的角度出发, 剖析桂西北高山汉民歌的发展现状。

关键词: 族群认同; 广西高山汉；民歌；文化认同

\section{1.高山汉民歌的社会功能}

高山汉民歌，是高山汉人民生活的一个缩影，它 承载着无数高山汉人民在迁徙中、在奔波中、在挣扎 中的文化记忆。高山汉民歌包括了高山汉人民的风土 人情、生产生活、历史变迁等内容, 因此民歌的影响 是非常广泛且深远的, 在高山汉族群文化的形成、维 持中具有特殊的功能。主要具有以下几个功能:

\section{1.文化传承功能}

“民歌是经过长期而广泛的群众性的即兴创作， 口头传唱而逐渐形成和发展起来的。”由于恶劣的居 住环境, 早年高山汉一直生活在高寒石山地区, 环境 相对来说比较封闭, 与外界的交流很少甚少, 教育水 平也相对有限。因此传承下来的民歌架起了一座高山 汉人民受教育的桥梁, 并将他们的智慧集中在创作民 歌上。他们不仅擅长通过唱歌传播丰富的知识, 而且 还通过倾听和学习这些记录本族群历史和文化的歌 曲, 来开拓生活和事业、启迪心智，使民间歌曲成为 
了文化传承的重要途径之一。如:

苦悠悠, 廊檐吃饭雨飘头;

牛在栏里叹大气, 牲畜为我叹苦愁。

苦丁丁，廊檐吃饭雨淋身;

牛在栏里叹大气, 牲畜为我叹苦情。

这是一首解放前的长工苦歌, 生动的记录了旧时 长工的悲惨生活。改革开放后, 尽管高山汉人民的生 活环境有了很大改善, 但唱民歌仍然是他们生活的一 部分。通过分析不同时期高山汉族民歌的内容创作， 我们可以发现高山汉人民在时代变迁中生存环境、生 活条件等的变化。如这首山歌中的歌词: “改革开放二 十年, 家乡旧貌变新颜; 楼房起到茅草岭, 麻雀难找 旧廊檐”。主要描述了改革开放以后的新政策改善了 广大高山汉人民生活环境, 人们自然纵情高歌起来。

传承了其独特文化记忆的高山汉民歌, 就像高山 汉人民的“百科全书”，仍然教育着一代又一代的高山 汉儿女。

\section{2. 道德教化功能}

音乐具有教育功能这一说法在中国古代文献中 被许多音乐家、文学家所提出过。早在周代, 统治者 就试图通过一套完善的“礼乐制度”对人的外部行为 和内部思想建立统一的规范。在《礼记·乐记》中还讨 论了“乐”与“德”之间的关系。春秋战国时期，孔子的 提出的“礼乐思想”也表明: “礼”与“乐”对于社会规划 和发展同样重要。高山汉族民歌文本融合了丰富的传 统文化, 例如时令节日、历史神话传说、浐语、歌谣 等。如:

正月想妹梅花红，二月想妹李花开；梅花谢了李 花谢, 不知哪时喜才来。

三月想妹清明时, 四月想妹种禾苗; 细雨过后禾 苗起，不见情妹心烦焦。

五月想妹䕃田草, 六月想妹插晚稻; 可能情妹丢 哥了, 十条肠子断九条。

七月想妹交白露, 八月乡妹想秋分; 森林落叶秋 风起, 妹你变心不变心。

九月想妹是秋收, 十月想妹路一条; 请妹连哥要 留心, 秋实结果要成桃。

此曲实际上是一首情歌, 但其中大量的穿插了高 山汉传统时令节日来说明时间的流逝, 将对阿妹的思 念之情与耕种劳动的进程相结合, 展现了高山汉人民 的聪明才智和丰富的传统文化，以音乐的形式来督促、 教育高山汉人民。再如:

正月犁秧田，二月修田基;

三月撒稻种, 四月插秩时。

五月祭田魂, 六月耘田去;
七月禾怀胎，八月穗出齐。

九月可开镰，十月湿谷到屋里；

十一月干谷进了仓, 腊月做糍给娘吃。

这首山歌主要阐述了高山汉人民栽种稻子的进 程, 按其叙述的从栽种到收获的季节来看, 是符合岭 南地区亚热带气候的栽种条件的, 展现了高山汉人民 辛勤劳作的场景。其次, 将收获了的谷穗做成糍粑送 给爹娘, 表达了高山汉儿女对父母的感恩之情。

民歌不仅可以帮助人们掌握时间更迭和生产劳 动的动态, 还可以帮助人们了解历史、铭记历史。高 山汉民歌朴素而亲切, 歌词大多生动地描述了高山汉 人民的辛勤工作、尊老爱幼、孝敬父母的点点滴滴， 是高山汉人民构建正确的人生观、价值观教育的一个 重要途径。

\section{3. 娱乐功能}

民歌的娱乐功能分为三种：自娱、娱人和娱神。 高山汉民歌也具有上述三个功能, 但自我娱乐是他们 的主要消遣方式。高山汉人民常常在劳作和休闲时唱 民歌, 主要用于缓解工作压力, 消除工作中的孤独感 或表达对家人、恋人的思念之情等等, 这都反映出民 歌的自我娱乐功能。如: “闷闷闲闲过一天, 打起火来 烧杆烟; 打起火来把烟烧, 人也精神嘴也甜。”这首山 歌生动的描述了一位闲来无事的老汉在家唱起山歌 的场景, 真实的记录了高山汉人民悠然、乐观的生活 情趣。

同时，高山汉民歌还具有人际交往和抒情表意的 娱人功能, 用歌曲、歌声来实现人与自然之间的交流、 沟通, 尤其是当社会条件不那么发达时, 民歌已成为 异性之间互动的重要工具。如:

男: 哥是天上白斑鸠, 妹是花园红石榴; 吃不饱 来看不饱, 给哥难舍又难丢。

女: 生要连来死要连, 不管爹妈在眼前; 不怕哥 嫂在身边, 深深恋哥一万年。

男: 牛角尖尖装芝麻, 生死要连这枝花; 刀子夺 命我不怕, 五马分尸也管他。

女: 桐子开花为桐油, 情妹挨打为风流; 打是打 在妹身上, 痛是痛在哥心头。

此曲描绘了一幅男女互相爱慕、誓死要在一起的 一幅画面, 表达了高山汉年轻男女对爱情的忠贞不渝。

另外, 还有不少用于民俗仪式中的民歌用来表达 对神的敬意和崇拜。

\section{4. 审美功能}

时代变迁, 高山汉民歌仍然不断传承和发展着, 并且成为了高山汉人民精神生活的重要支柱。它具有 深刻的美学意义, 其音乐审美符合传统汉族审美标准。 
主要表现在两个方面, 分别是内容之美和形式之美。

首先，在民歌的内容上，它的的唱词、音韵、顿 挫、语句节奏，都代表着高山汉人民的的精神气质和 审美标准。其中, 情歌是所有题材中曲目数量最多的, 也是最具代表性的。如:

男: 月亮里面有桂枝, 情哥早想摘一支; 鲁班师 傅死的早，无人帮哥搭天梯。

女: 眼看表哥身穿绿, 手里提着半斤肉; 心想同 哥打平伙，可惜人生面不熟。

男: 妹是园中一枝梅, 哥今刨土来栽培; 还望老 天多下雨，成双就看这一回。

女: 三匹芭茅搭座桥, 两匹落地一匹飘; 只要真 心又诚意, 茅草更比钢丝牢。

男女对唱之间你来我往矜持的语言反映了高山 汉人朴素委婉的表情方式, 表达了高山汉人民对美好 生活的期待。从以上民歌文本中可以反映出高山汉人 民关于非音乐性内容所构建的绘画性原则, 这是人们 长期对生活保持细微的、具有深度的观察而形成的审 美能力。

另外, 高山汉民歌的形式美主要表现在两个方面: 一是旋律美。通过笔者之前对高山汉五类民歌体裁的 音乐本体分析, 可以得知它的旋律多为五声徵调式, 如《新娘敬茶散鞋歌》《十叹父母养育恩》《蛤蟆调》 等曲目均为五声徵调式, 这与我国南方汉族民歌相似。 旋律走向多呈波浪起伏居多, 旋律起伏较小, 旋律进 行常以级进的大二度为主, 其中穿插小三度音程, 因 此曲调比较舒缓。有悠长婉转的山歌, 也有朗朗上口 的小调; 也有类似念诵型的真挚感人的风俗歌; 还有 天真活泼的儿歌。

二是织体美。高山汉民歌中的山歌经常使用各种 装饰音和固定拖腔, 同时歌手在即兴歌唱时根据情绪 和情感的抒发常在句末加花, 使高山汉民歌的音乐更 加丰富多彩。其中, 还常常使用一音多润腔的装饰手 法, 即一个音前、后同时运用装饰音, 这在高山汉民 歌中并不常见，

对这种民歌美的认同和审美情感之来源是其将 感知、理解、想象、认知等心理因素融合成一种独特 的活动形式, 以可感觉、可触摸的形象或声音来反映 现实、再现现实, 并以此提高人们从生活中认识、把 握美的能力, 其后融入进音乐中, 再以音乐的形式显 现出来, 表达了高山汉人民意识深层的审美特征。

综上, 高山汉民歌承载了高山汉人民的人文历史、 民俗风情、审美情操, 因此它具有很强的社会功能, 对高山汉人民的文化传承、价值观建构影响深厚。

\section{2.高山汉民歌族群文化认同}

如何解释生活在不同区域、来自不同地方的人却 拥有共同的文化特质，尤其是风俗习惯、住房饮食、
语言服饰都存在着大同小异的特点。高山汉是否属于 族群? 如果是, 族群文化认同体现在哪些方面? 那么 本节通过探讨高山汉的族群归属问题以及其族群文 化认同的体现，旨在说明高山汉民歌生成、保存过程 的主要影响因素。

“认同”一词的最早来源于心理学的概念, 是由奥 地利著名精神病医生、心理学家弗洛伊德于 19 世纪 末提出的。他认为认同主要指的是是个人或群体在感 情上或心理上趋同的一个过程, 对于个人或群体心理 发展的影响进程中, 其认同的过程显示出长期性、稳 定性的特征。其后, 20 世纪 50 年代美国精神病学家、 发展心理学家、精神分析学家埃里克森在他撰写的著 作《认同与生命周期》（Identity and life Cycle）中也提 出了“认同”的概念。《族群认同、族群认同的发展及测 定与研究方法》一文中还指出“族群认同”（ethnic group identity）包括自我认同、归属感、对参照群体 的认知以及个体在群体中的价值分享等不同的维度, 是动态的多维度的结构。而广义的族群认同不仅包括 个体对本族群的信念、态度和参与行为, 而且还包括 个体对他族群的信念、态度和参与行为。以上表明, 在影响族群认同建构的诸多要素中, 文化要素被视为 最重要的客观要素。自 1980 年以来, 美国众多民族 音乐学家开始关注“音乐与认同”之间的关系。早期的 研究人员包括克里斯·沃特曼 (Chris Waterman)、曼努 埃尔·佩纳 (Manuel Pena)、戈登·汤普森 (Gordon Thompson）等等。纵众多相关文献著作, 研究者们反 复强调音乐有助于建构社会认同, 音乐在族群边界认 同形成与维护中如何发挥重要作用以及新型的音乐 形式如何在建构新兴的身份认同中发挥作用。近年, 许多中国音乐研究者开始关注到“音乐与认同”这一 主题, 如湖南师范大学音乐学院硕士研究生导师赵书 峰教授发表于《中央音乐学报》 2017 年第二期的论文

《族群边界与音乐认同—冀北丰宁满族“吵子会”音 乐的人了些阐释》以及发表在《中国音乐》2018 年第 1 期的论文《移动的边界、身份的变色龙一冀北丰宁 “吵子会”音乐认同变迁的历史人类学研究》等。随着 全球化的发展, 所有媒体和互联网技术的迅速普及, 不同国家、民族、族群和区域之间的联系越来越紧密, 相互之间不同音乐文化的交流、互动、融合与冲突变 得越来越普遍。不同音乐文化之间最根本的冲突是音 乐认同的融合和碰撞。音乐既带有人的个体属性、社 会属性和文化属性, 又来自于人的创造性思维和发散 性思维。高山汉民歌即为异地、同族之间进行传播的 音乐文化认同现象，是历史与当下之间搭建起的一架 文化认同的桥梁，具体表现在风俗习惯、民间信仰等 方面。

\section{1. 风俗习惯}

风俗习惯对族群而言, 是指某族群在长期的历史 发展过程中逐渐形成的具有稳定性、持续性的的传统 习俗。

据笔者的走访调查和收集的文献资料中所记载, 
高山汉迁入桂长达百余年，周围居住着壮、苗、瑶、 仡佬族等少数民族，但与他们之间的文化交流却没有 太多，生活、生产习惯以及人生礼俗等风俗习惯与传 统汉族基本相同。

首先, 广西高山汉与传统汉族的住房结构基本相 同，在隆林县隆或乡、介廷乡以及田林县、凌云县等 高山汉聚居地, 很清晰的就可以分辨出高山汉居住的 房屋。他们的住房基本都为平房，正厅的中间墙壁上 中间设有祖先神位，悬挂“天地君亲师”，用以祭拜先 祖神灵; 正厅右侧为厨房, 厨房放置火铺, 用以烧水、 取暖、煮饭, 同时进出屋子大都为右侧的小门; 左侧 为卧室, 正厅大门及侧门基本不开。随着经济的快速 发展, 很多高山汉搬到了县里或者生存更适合居住的 平地, 新的楼房很少在造火铺, 比较常见的是楼层数 量从一层增加至两层楼, 但整体构造基本与上述无大 差别。

在婚丧嫁娶、修房生育等习俗上也普遍相同。例 如结婚当天, 新娘出嫁时、宴请宾客时要唱哭嫁歌、 敬酒歌等; 伴郎还要与伴娘一方的人进行对歌。如今, 高山汉人民的婚礼仪式中很少出现对歌, 只有少数热 爱民歌的人家会在婚嫁仪式前夕或者婚礼当天邀请 一些山歌艺人来助兴。

\section{2. 民间信仰}

“在人类所能支配的一切力量中, 信仰的力量最 为惊人。”民间信仰不仅是高山汉精神文化的重要组 成部分, 而且是体现高山汉族群建构的纽带。民间信 仰深深扎根于乡土社会中, 以万物有灵为主体。桂西 北高山汉崇拜的神灵主要有祖先神、自然神和鬼神等 等, 具体的有祖先, 灶王神, 土地神, 山神和观音等。

高山汉人民与传统西南地区的汉族人一样, 在众 多神灵崇拜中, 供奉着最为重要、最频繁的即为祖先 神。几乎所有的高山汉人民居住的房屋都会在正厅中 供奉祖先神, 甚至因原籍来自相同地方且姓氏相同而 聚居在一起的高山汉还建立了祠堂来祭拜祖先。农历 的初一、十五以及节日时期, 都会以香烛、新鲜的瓜 果点心祭祀祖先。除祖先神之外, 祭祀比较多的是自 然神, 在新年之始, 为祈求来年丰收平安, 有的还带 上香纸、酒、果品到附近的土地庙、山神庙、观音庙 或者传说中有灵气的水井、桥、神树旁进行祭拜。

高山汉在文化特质上的维持和认同促进了高山 汉民歌的形成，而高山汉民歌以其生活性、口头性的 基本特性渗透于高山汉的生活当中, 是族群建构中最 为显现的象征符号。

另外要说明的是高山汉族群文化认同维持主要 有两个解释: 第一为原生论, 主要指的是基于共同的 语言属性、宗教信仰、种类族别、族别属性以及共同 居住下领土的“原生纽带”等因素是族群成员相互关联 的重要因素, 同时十分强调相同的语言属性、宗教信 仰、种类族别、族别属性以及共同生活领土是整个人
类历史上最基本的社会组织原则, 而且这样的原则存 在于一切人类团体中, 并超越时空而存在。因此对族 群成员来说, 原生性的纽带和情感是根深蒂固和非理 性、潜意识的。那么这就能解释当我在采访高山汉的 艺人或者相关音乐文化爱好者时，他们都会提到: “我 们都很喜爱唱山歌, 这都是老祖宗留下来的东西”。让 我记忆深刻的是一位嫁到瑶寨生活了二十多年高山 汉妇女高月琴, 她是经人介绍然后嫁到浪平乡平山街 管辖下的一个屯, 里面 $95 \%$ 以上的都是瑶族人。我去 采访她时她正在田间劳作，当我提出想问她一些问题 以及想录一段她唱歌的视频时，她十分差怯说道类似 “我只会讲汉话, 我可以听懂瑶话, 但我不会说”“平常 我很喜欢唱汉歌, 几乎每天都要唱山歌 (民歌)”。由 此得知, 这种原生性的血缘性的纽带是非常根深蒂固 的。高山汉民歌的传播就是在基于人心理下意识的表 达以维持这个认同纽带。

第二指的是对立论, 对立论的前提需要说明的是 不同族群之间对立的过程是族群认同的得以维持的 主要原因。提出的核心观点即为“持久的认同体系”和 “对立过程”。在这个“对立过程”中隐藏的是一方族群 对另一族群的文化的吞并, 那么在高山汉与其他族群 相处的过程中，类似“火铺”等带有被迫意味的习俗似 乎带有了一些对本地人眼中“异文化”控制, 此时以语 言、宗教、习俗、民歌等为依托来主动的进行实现群 体目标。继而持久的认同体系在其对立过程中, 本文 化成功抵御异文化的同化而存留下来, 族群认同在时 间的前进中得以维持，丰富多彩的汉族民歌文化得以 保存。

\section{3.结论}

自明末清初始, 大量的西南移民进入桂西北这片 神秘的高山时, 富有地域特色鬼米力的民歌开始流传开 来，吸引了着越来越多的人为之驻足。高山汉民歌在 不同区域、不同时空中继续得以传播，除了拥有共同 的文化习俗、民间信仰等建构族群的重要因素外，民 歌里使用的旋律节奏、调式音列等相似的音乐形态特 点是其拥有共同的审美造就的，是对自身传统文化的 认可和保存, 而民歌则是其族群文化认同中最为显现 的象征符号。高山汉民歌的传播形式并不是一个特别 的音乐文化传播现象，它是同一民族之间文化交流、 文化深度认同的一个缩影，是传统汉族民歌传播的普 遍现象，也是处于少数民族中的汉族相互交流、沟通 的纽带。

\section{REFERENCES}

[1] Wu Hepei, Luo Zhifa, Huang Jiaxin. Exploring the Secrets of the Clan Islands'Langping Gaoshan Han[M]. Nanning: Guangxi Nationalities Publishing House, 1999.

[2] General History Museum of Guangxi Zhuang Autonomous Region. Twenty-four History of 
Guangxi Records [M]. Nanning: Guangxi People's Publishing House, 1989.

[3] Huang Yunzhen. The historical migration of the Han population and the color pattern of southern Han folk songs[J]. Chinese Musicology, 1989(4):36-48.

[4] Wu Yongsheng, Feng Jian, Zhang Xiaolin. The Regional Features and Geographical Basis of Chinese Folk Song Culture[J]. Human Geography, 2005, 20(2): 88-92.

[5] Zhao Shufeng. Ethnic Boundary and Music Identity: An Anthropological Interpretation of the "Baizihui" Music of the Manchu in Fengning, Northern Hebei Province[J]. Journal of the Central Conservatory of Music (2): 43-48.

[6] Timothy Rice, Wei Linlin. Reflection on Music and Identity in Ethnomusicology[J]. Music Exploration, 000(1):49-58.

[7] Jiang Mingtun: "An Introduction to Han Folk Songs", [M]. Shanghai: Shanghai Music Publishing House, 2004.

[8] Huang Zongdao, Zhou Wuhong: "Leye Folk Songs (1)", [M]. Beijing: China Radio and Television Press, 2008.

[9] Che Wenbo: Selections of Freudian Principles. Liaoning People's Publishing House, 1998, p. 375.

[10] Wan Minggang, Wang Zhou: Ethnic Identity, the Development and Measurement and Research Methods of Ethnic Identity. World Nation. 2007 (3). p1-9.

[11] Gustave Le Pen, writing. Feng Keli, translation: the rabble: a study of popular psychology [M]. Beijing: Central Compilation and Translation Publishing House, 2004. (p97).

[12] Li Xiangfu: Relevant concepts and basic theories in ethnic research. Journal of Guangxi University for Nationalities, 2009 (9). 